\title{
Extraction and recovery of toxic acidic components from highly acidic oil using ionic liquids
}

\begin{abstract}
Naphthenic acid (NA) is a toxic compound that exists in the effluent discharged from highly acidic oil refineries. The amount of NA present in acidic crude oil can be as high as $4 \mathrm{wt} \%$. The complicated structure of NA poses a challenge for oil refineries in their effort to extract NA from the heavy crude oil in an economical and environmental friendly manner. In the current study the extraction of NA from highly acidic model oil by ionic liquid (ILs) was performed using 1,8-diazobicycloundec-7-ene (DBU) based cation in combination with the thiocyanate anion. A detailed computer simulation study on the mechanism of NA extraction by the ILs was also performed. The extracted NA was completely recovered and the ILs used were regenerated by simple addition of water. It was found that increasing of the alkyl chain length increases the percent NA removal. Computer simulation suggests those thiocyanate anions are found to be playing a major role in the NA extraction process.
\end{abstract}

Keyword: Ionic liquids; Naphthenic acid; Thiocyanate anion; Regeneration; Extraction 